\title{
Sudden gains in Cognitive Therapy and Interpersonal Psychotherapy for adult depression
}

Citation for published version (APA):

Lemmens, L. H. J. M., DeRubeis, R. J., Arntz, A., Peeters, F., \& Huibers, M. J. H. (2016). Sudden gains in Cognitive Therapy and Interpersonal Psychotherapy for adult depression. Behaviour Research and Therapy, 77, 170-176. https://doi.org/10.1016/j.brat.2015.12.014

Document status and date:

Published: 01/02/2016

DOI:

10.1016/j.brat.2015.12.014

Document Version:

Publisher's PDF, also known as Version of record

Document license:

Taverne

\section{Please check the document version of this publication:}

- A submitted manuscript is the version of the article upon submission and before peer-review. There can be important differences between the submitted version and the official published version of record.

People interested in the research are advised to contact the author for the final version of the publication, or visit the DOI to the publisher's website.

- The final author version and the galley proof are versions of the publication after peer review.

- The final published version features the final layout of the paper including the volume, issue and page numbers.

Link to publication

\footnotetext{
General rights rights.

- You may freely distribute the URL identifying the publication in the public portal. please follow below link for the End User Agreement:

www.umlib.nl/taverne-license

Take down policy

If you believe that this document breaches copyright please contact us at:

repository@maastrichtuniversity.nl

providing details and we will investigate your claim.
}

Copyright and moral rights for the publications made accessible in the public portal are retained by the authors and/or other copyright owners and it is a condition of accessing publications that users recognise and abide by the legal requirements associated with these

- Users may download and print one copy of any publication from the public portal for the purpose of private study or research.

- You may not further distribute the material or use it for any profit-making activity or commercial gain

If the publication is distributed under the terms of Article $25 \mathrm{fa}$ of the Dutch Copyright Act, indicated by the "Taverne" license above, 


\title{
Sudden gains in Cognitive Therapy and Interpersonal Psychotherapy for adult depression
}

\author{
Lotte H.J.M. Lemmens ${ }^{\mathrm{a},{ }^{*}}$, Robert J. DeRubeis ${ }^{\mathrm{b}}$, Arnoud Arntz ${ }^{\mathrm{a}, \mathrm{c}}$, Frenk P.M.L. Peeters ${ }^{\mathrm{d}}$, \\ Marcus J.H. Huibers a, b, e
}

a Department of Clinical Psychological Science, Maastricht University, P.O. Box 616, 6200 MD Maastricht, The Netherlands

b Department of Psychology, University of Pennsylvania, 3720 Walnut Street, Philadelphia, PA 19104-6241, USA

${ }^{c}$ Department of Clinical Psychology, University of Amsterdam, P.O. Box 19268, 1000 GG Amsterdam, The Netherlands

d Department of Psychiatry and Psychology, Maastricht University, P.O. Box 616, 6200 MD, Maastricht, The Netherlands

e Department of Clinical Psychology, VU University Amsterdam, Van der Boechorststraat 1, 1081 BT Amsterdam, The Netherlands

\section{A R T I C L E I N F O}

\section{Article history:}

Received 8 June 2015

Received in revised form

15 December 2015

Accepted 20 December 2015

Available online 23 December 2015

\section{Keywords:}

Sudden gains

Cognitive Therapy

Interpersonal Psychotherapy

Depression

RCT

\begin{abstract}
A B S T R A C T
Objective: We examined the rates, baseline predictors and clinical impact of sudden gains in a randomized comparison of individual Cognitive Therapy (CT) and Interpersonal Psychotherapy (IPT) for adult depression.

Method: 117 depressed outpatients received 16-20 sessions of either CT or IPT. Session-by-session symptom severity was assessed using the BDI-II. Sudden gains were examined using the original criteria as defined by Tang and DeRubeis (1999b). Furthermore, we examined whether the duration of the between-session interval at which sudden gains were recorded affected the results.

Results: There were significantly more patients with sudden gains in CT (42.2\%) as compared to IPT (24.5\%). The difference appeared to be driven by the criterion representing the stability of the gain. No between-group differences were found with regard to the magnitude, timing and predictors of the gains. Those with sudden gains were less depressed at post-treatment and follow-up. After controlling for the duration of the between-session interval, the difference in rates between the two conditions became a non-significant trend. Other sudden gains characteristics were similar to those observed when allowing for longer intervals as well.

Conclusions: The current study indicates differences in occurrence of sudden gains in two treatment modalities that overall showed similar results, which might reflect different mechanisms of change.
\end{abstract}

(c) 2016 Elsevier Ltd. All rights reserved.
For a long time in investigations of the course of change during psychotherapy it had been assumed that the typical trajectory of change was gradual and linear (Kazdin \& Nock, 2003; Kraemer, Wilson, Fairburn, \& Agras, 2002; Laurenceau, Hayes, \& Feldman, 2007). However, studies of the temporal course of change during treatment have revealed that a substantial portion of a patient's total reduction in symptom intensity often occurs suddenly, rather than gradually over the course of treatment. Analyses of individual change patterns in two large trials of Cognitive Therapy (CT) for depression (Elkin, Shea, Watkins, \& Imber, 1989; Hollon et al., 1992) showed that almost $50 \%$ of the patients experienced half of their total symptom improvement between two consecutive therapy

\footnotetext{
* Corresponding author

E-mail address: Lotte.Lemmens@Maastrichtuniversity.nl (L.H.J.M. Lemmens).
}

sessions (Tang \& DeRubeis, 1999a). These large improvements in symptoms during a single between-session interval were termed "sudden gains" (Tang \& DeRubeis, 1999b). In the initial study that described this phenomenon, Tang and DeRubeis found that patients who experienced sudden gains showed more therapy improvement and had higher rates of recovery compared to those without sudden gains, and they remained so 18 months later. Further research showed that patients with sudden gains were up to $75 \%$ less likely to experience relapse and recurrence in the 24 months after treatment termination (Tang, DeRubeis, Hollon, Amsterdam, \& Shelton, 2007). The finding that sudden gains during treatment are associated with more favorable treatment outcomes, both in the short run and in the long term, has important implications for the prognosis of depression, given that for many patients it can follow a chronic or recurrent course.

Since its introduction in 1999, sudden gains have been 
examined in at least 10 studies, covering at least 7 forms of psychotherapy for depression (see meta-analysis of Aderka, Nickerson, Bøe, \& Hofmann, 2011 for an overview). In the six studies that focused on individual CT for adult depression, sudden gain rates ranged from $33 \%$ to $45 \%$. In each study, those with sudden gains showed lower levels of depressive symptomatology at posttreatment and/or follow-up as compared with those who did not experience sudden gains (Busch, Kanter, Landes, \& Kohlenberg, 2006; Hardy et al., 2005; Tang \& DeRubeis, 1999b; Tang, DeRubeis, Beberman, \& Pham, 2005; Tang et al., 2007; Vittengl, Clark, \& Jarrett, 2005). It should be noted, however, that the criteria for identifying sudden gains differed across the studies, making between-study comparisons of the rates problematic.

Interpersonal Psychotherapy (IPT) is, next to CT, the most commonly practiced and extensively researched psychotherapy for depression (Cuijpers et al., 2011; Hollon, Thase, \& Markowitz, 2002). Kelly, Cyranowski, and Frank (2007) examined the occurrence and impact of sudden gains during IPT in female patients with recurrent depression. They observed sudden gains at a similar rate as has been reported in CT (34\%), but found no evidence that sudden gains were associated with better outcomes at posttreatment or during follow-up. This is, to our knowledge, the only study of sudden gains during IPT for depression.

Furthermore, patient pre-treatment characteristics that distinguish those who will go on to have sudden gains from those who will not, have only been explored in a few studies in the context of CT and Behavioral Activation. In these studies, variation in depressive symptom severity, and measures of negative cognitions and interpersonal functioning at baseline have not been found to predict the occurrence of sudden gains (Hunnicutt-Ferguson, Hoxha, \& Gollan, 2012; Kelly, Roberts, \& Ciesla, 2005; Vitteng] et al., 2005). The pre-treatment characteristics that might predict sudden gains in IPT however, have yet to be examined, as has the possibility that different characteristics would be associated with sudden gains in CT versus IPT.

The aim of the present study was to replicate and extend previous research on sudden gains in individual psychotherapy for adult depression. Data come from a recently published randomized comparison of CT and IPT for depression in which we found no significant differences on outcome in the acute phase (Lemmens et al., 2015). We identified the patients who met criteria for sudden gains and examined the relation between sudden gain status and end of treatment symptom severity, as well as symptom severity at five-months follow-up. In addition, we explored baseline patient characteristics that might predict the occurrence of sudden gains, both within each treatment as well as across the two treatments. In a series of secondary analyses, we examined whether the duration of the between-session interval at which sudden gains were recorded affected the results obtained.

We expected that sudden gains would appear in both CT and IPT at a similar rate as in other studies of treatment for depression (approximately 40\%: Busch et al., 2006; Hardy et al., 2005; Kelly et al., 2007; Tang \& DeRubeis, 1999b; Tang et al., 2005; Tang et al., 2007; Vittengl et al., 2005). Also in line with previous research, we expected that individuals who experienced sudden gains would experience superior outcome, both at treatment termination and through the follow-up period (Tang \& DeRubeis, 1999b; Tang et al., 2007). Given the exploratory nature of our predictor analysis, there were no clear a priori hypotheses regarding the pre-treatment factors associated with sudden gains. In general, we expected that lower levels of depressive symptomatology and dysfunctional processes would be predictive of sudden gains. Previous research on how CT and IPT for depression compare in terms of frequency, magnitude, timing, and predictors of sudden gains is lacking. However, analogous to work on sudden gains in CT versus IPT in the field of social anxiety disorder (Bohn, Aderka, Schreiber, Stangier, \& Hofmann, 2013) one would expect no differences between the two conditions.

\section{Method}

\subsection{Data source}

The study sample consisted of 151 adult outpatients, aged 18-65, who participated in an RCT examining the effectiveness and mechanisms of change of individual CT $(n=76)$ and IPT $(n=75)$ for depression (Lemmens et al., 2011). ${ }^{1}$ Participants' primary diagnosis was Major Depressive Disorder, as determined by the Structured Clinical Interview for DSM-IV Axis I Disorders (SCID-I; First, Spitzer, Gibbon, \& Williams, 1997). Potential participants were excluded if they were currently taking antidepressant medication, if the duration of their current episode of depression exceeded 5 years, and/or if they met criteria for Bipolar Disorder, Substance Abuse or Dependence, or Mental Retardation (IQ < 80). All participants provided written informed consent before entering the study. Treatment consisted of individual sessions, each lasting $45 \mathrm{~min}$. Depending on the patient's progress, 16 to 20 sessions were provided. The CT protocol followed Beck, Rush, Shaw, and Emery (1979). The IPT protocol was based on Klerman, Weissman, Rounsaville, and Chevron's (1984) manual. The primary outcome measure, the Beck Depression Inventory-II (BDI-II: Beck, Steer, \& Brown, 1996), was completed by patients immediately prior to each therapy session, at the end of the treatment phase ( 7 months), and monthly throughout the 5 month follow-up period (8-12 months). Further details concerning the design of the study, measures, interventions, and participants flow can be found in Lemmens et al. (2011; 2015). The Medical Ethics Committee of Maastricht University approved the study protocol. The study is registered at the Dutch Cochrane Centre through the Netherlands Trial Registry (ISRCTN67561918).

\subsection{Data preparation}

Similar to Tang and DeRubeis (1999b), we included for the purpose of examining sudden gains only those patients who received 8 or more sessions of therapy and whose BDI-II score at the first session was available, and was 15 or higher. Of the 76 CT patients, 12 were excluded ( 6 received fewer than 8 sessions, 2 had BDI-II scores that were missing, and 5 had BDI-II scores that were lower than 15 at the first session). In the IPT group, 23 out of 75 patients were excluded ( 9 received fewer than 8 sessions, 4 had missing BDI-II scores, and 10 had a first-session BDI-II score lower than 15). As some patients were excluded for more than one reason, this resulted in sample sizes of 64 in the CT condition and 53 in the IPT condition.

Patients in this sample ranged in age from 18 to $63(\mathrm{M}=41.8$, $\mathrm{SD}=12.1$ ). Two-thirds $(66.7 \%$ ) were female, $59.8 \%$ were educated at intermediate vocational level (lower: 18.8\%, higher: $21.4 \%$ ), and $60.7 \%$ were married or in a committed relationship. The average score on the BDI-II at baseline was $30.6(\mathrm{SD}=8.5)$, and $47.0 \%$ of the sample was diagnosed with recurrent depression. More than half of the patients (58.1\%) suffered from severe depression (BDI-II score $>29$; Beck et al., 1996) but the majority (59.0\%) was still actively employed. The 34 patients who were excluded from

\footnotetext{
1 As outlined in Lemmens et al. (2011), the full design also includes a third arm; a 2-month waiting list control (WLC) condition followed by treatment of choice $(n=31)$. However, for the purpose of the present paper, we focused our attention only on sudden gains occurring in the two active conditions CT and IPT.
} 
analyses did not differ significantly from those who were included on any of the baseline variables described above. As expected, significant differences were found on variables that were used to exclude patients from the study: mean number of sessions atten$\operatorname{ded}(10.0(S D=7.0)$ vs. $15.9(S D=3.7))$ and mean first session BDI-II score $(20.6(S D=12.4)$ vs. $29.2(S D=8.0))$.

\subsection{Statistical analyses}

A total of 1667 between-session intervals were analyzed for identification of sudden gains using the original criteria of Tang and DeRubeis (1999b). The sudden gain must be $(a)$ large in absolute magnitude (at least 7 BDI-II points), (b) large in relative magnitude (representing at least $25 \%$ of the pre-gain session's BDI-II score) and (c) large relative to symptom fluctuation (significantly higher mean in the three pre-gain sessions than in the three post-gain sessions in order to rule out random fluctuation in treatment). ${ }^{2}$ As in previous studies, reversals were defined as having occurred whenever a patient gave up $50 \%$ of the symptom improvement that constituted the sudden gain (Tang \& DeRubeis, 1999b). We mapped out rates and characteristics of sudden gains and reversals, and compared baseline sociodemographic characteristics and pretreatment clinical measures of patients with and without sudden gains. Furthermore, differences between the two interventions were examined.

In order to explore the predictive value of baseline characteristics on the occurrence of sudden gains during treatment, using univariate logistic regressions we identified baseline variables with promising associations with the dependent variable $(p<.20)$. The variable measuring the presence or absence of one or more sudden gains (gain) was the dependent variable. As potential predictors we selected several demographic variables (gender, age, marital status, level of education, employment status), depression specifiers (BDIII, QIDS, and BHS, first or recurrent depression), measures of general functioning and psychological distress (BSI, EuroQol, RAND-36), and the presence of Axis I and/or II comorbidity. Measures of cognitive processes (DAS-A-NL, LEIDS, RRS-NL, and ASQ assessing dysfunctional attitudes, cognitive reactivity, rumination, and attributional style respectively) were also included, as were measures of interpersonal functioning (IIP) and self-esteem (SLSC-R). A complete listing of these measures and their subscales can be found in Data Supplement I. Full descriptions of the measures are reported elsewhere (Lemmens et al., 2011). To examine the exact effects of these potential predictors on outcome, the selected variables were entered in a multivariate regression model and were eliminated in a manual backward procedure until the model consisted only of significant predictors $(p<.05)$.

To determine whether patients with sudden gains benefitted more from treatment as compared to patients without sudden gains, we conducted a series of analyses of covariance (ANCOVAs), with sudden gain (present vs. absent) as the independent variable and BDI-II scores (separate analyses at 7 months and at 12 months) as the dependent variables. Pre-treatment severity (BDI-II baseline)

\footnotetext{
2 The $c$ criterion requires at least two BDI-II data points before and after the symptom changes. This automatically meant that large symptom changes that occurred very early or very late in treatment (within two sessions of initiating or terminating treatment) were excluded from further analysis. However, as outlined by Tang and DeRubeis (1999b) the first session is very different in nature from other sessions and therefore first-session sudden gains might be different phenomena than other sudden gains and should be treated as such (see Tang \& DeRubeis, 1999a,b for a detailed description of differences). Aderka et al. (2011) found further support for this notion by demonstrating that including these first-session sudden gains did not affect effect sizes of sudden gains. This line of reasoning can also be applied for the final treatment session.
}

was included as a covariate in all analyses. In an additional model at 12 months we controlled for post-treatment BDI-II score as well. In order to examine the differential effects of sudden gains on outcome for CT vs. IPT, we added condition and the interaction of gain*condition to the models. To ensure that patients who experienced sudden gains were not simply treatment responders, whereas patients without sudden gains did not respond to treatment, we selected only those patients whose BDI-II score decreased by at least $9 \mathrm{BDI}-\mathrm{II}$ points over the course of treatment and reran all models in this subsample.

We note that the original sudden gain criteria do not include a specification of the maximum duration of the between-session interval and therefore do not ensure the suddenness of the sudden gain. This was not a problem in the original studies of Tang and colleagues (1999b; 2005; 2007), since therapy sessions took place once or twice per week. As a result, the between-session intervals in those studies were less than or equal to 7 days. However, in the Dutch healthcare system and therefore in our trial, sessions are offered weekly, with the possibility to lower frequency toward the end of treatment. This can result in longer between-session intervals such that in some instances, decreases in symptoms noted between two consecutive sessions should not be considered 'sudden'. We therefore performed a series of secondary analyses in which we examined the effects of the duration of the betweensession interval. More specifically, we excluded all betweensession intervals longer than 14 days (the most common alternative to weekly sessions in the Netherlands) and reexamined frequencies, characteristics and baseline predictors of sudden gains in both conditions. Furthermore, to examine whether sudden gains that occurred within or outside this 14-day interval were differentially associated with outcome, we repeated the analyses of covariance, but with gain as three-level variable (no sudden gain, sudden gain within 14 day interval, sudden gain outside interval), and inspected the contrast of sudden gains within vs. sudden gains outside the 14 day interval.

\section{Results}

\subsection{Frequency and characteristics of sudden gains}

A total of 27 patients (42.2\%) in CT and $13(24.5 \%)$ in IPT ${ }^{3}$ met the full set of sudden gains criteria (see Table 1). There were no significant differences on baseline sociodemographic characteristics and pre-treatment clinical measures between those who were and were not identified as having experienced a sudden gain (see Table 2).

The difference in the proportion of sudden gainers in CT versus IPT was significant $\left(\chi^{2}(1)=4.02, p=.045\right)$. There were no differences between the two conditions with regard to the magnitude and timing of the sudden gains. The majority of sudden gain patients (70.0\%) had only one sudden gain (19 in CT; 9 in IPT); the others experienced two sudden gains. ${ }^{4}$ The average magnitude of the gain for the total sample was $11.0 \mathrm{BDI}-\mathrm{II}$ points $(\mathrm{SD}=4.3)$ and the median pre-gain session was 9 (see Table 3 for results stratified per condition). Of the 40 patients who experienced sudden gains, 8 (20.0\%) experienced a reversal before the end of therapy (6 in CT, 2 in IPT; see Table 1). Thus, the percentage of patients who

\footnotetext{
${ }^{3}$ Following Kelly et al. (2007) we also examined the occurrence of sudden gains in IPT in recurrently depressed women only $(n=18)$ and found that $22.2 \%$ of this subsample $(n=4)$ met the full set of criteria for sudden gains. In the CT condition, $13(48.1 \%)$ out of the 27 recurrently depressed women experienced one or more sudden gains.

${ }^{4}$ Of the 12 patients with two sudden gains, 4 ( 3 in CT and 1 in IPT) experienced a reversal between the two sudden gains.
} 
Table 1

Frequency of Sudden Gains and Reversal rates stratified according to treatment condition $(n=117)$.

\begin{tabular}{|c|c|c|c|c|}
\hline & \multirow{2}{*}{$\frac{\text { Large drop }(a \& b)}{n \text { patients }(\%)}$} & \multicolumn{2}{|c|}{ Sudden Gains $(a, b, \& c)$} & \multirow{2}{*}{$\frac{\text { Reversals }}{n \text { patients (\%) }}$} \\
\hline & & $n$ gains & $n$ patients $(\%)$ & \\
\hline $\mathrm{CT}(n=64)$ & $43(67.2)$ & 35 & $27(42.2)$ & $6(22.2)$ \\
\hline IPT $(n=53)$ & $40(75.5)$ & 17 & $13(24.5)$ & $2(15.4)$ \\
\hline
\end{tabular}

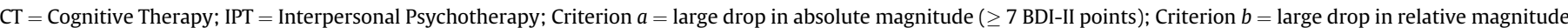

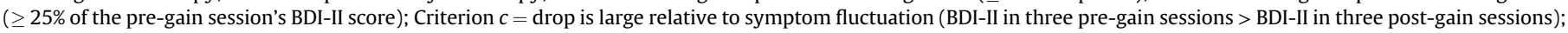
Reversal = giving up $50 \%$ of the symptom improvement that constituted the gain

Table 2

Sociodemographic characteristics and pre-treatment clinical measures for patients with and without sudden gains.

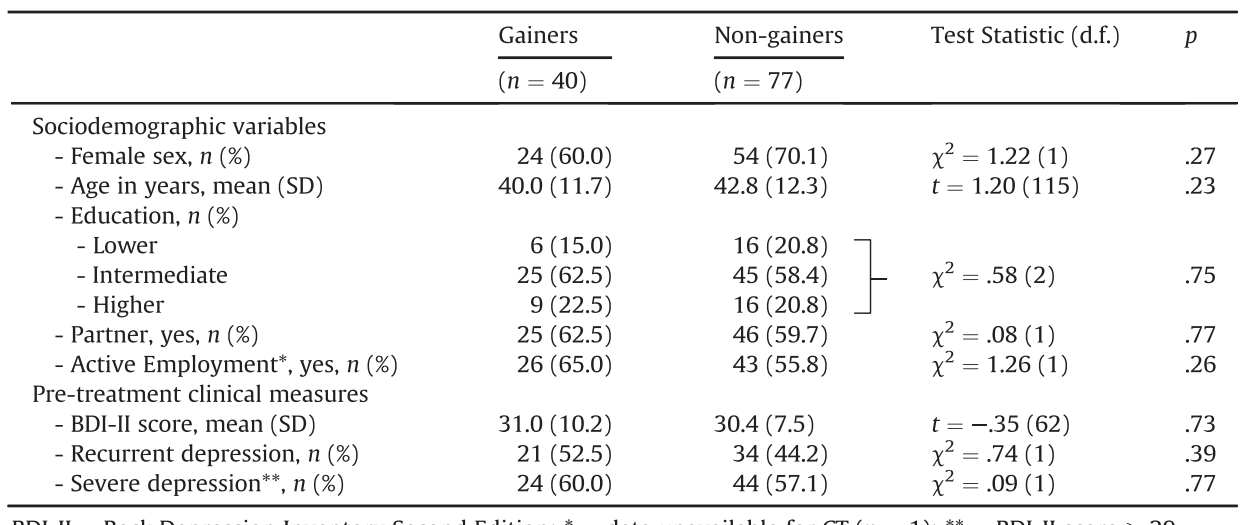

BDI-II = Beck Depression Inventory Second Edition; ${ }^{*}=$ data unavailable for CT $(n=1) ;{ }^{* *}=$ BDI-II score $\geq 29$.

experienced one or two sudden gains and did not experience a reversal of their sudden gain(s) was $32.8 \%$ in CT and $20.8 \%$ in IPT (see Table 3).

\subsection{A closer look at the differences between CT and IPT}

Given that the number of CT patients with sudden gains was twice what was observed in IPT, we went on to compare the rates of the individual sudden gain criteria between the two conditions. We began by comparing the number of patients who did not meet the criteria for eligibility for sudden gain analyses in this study (BDI-II at first session $<15$, or $n$ sessions $<8$ ). Significantly more IPT patients were excluded on these grounds (22/75 in IPT vs. 12/76 in CT; $\left.\chi^{2}(1)=3.97, p=.046\right)$. Thus, only 53 IPT patients, compared to 64 CT patients, were included in the sudden gain analyses. Nonetheless, the absolute numbers of patients who met sudden gain criteria $a$ and $b$, which index the magnitude of symptom improvement, were nearly identical in the two conditions (43 in CT vs. 40 in IPT; see Table 1). Differences between the conditions emerged only when criterion $c$, which indexes the stability of the drop, was applied. In CT, $62.8 \%$ of patients who met criteria $a$ and $b$ also met criterion $c$, whereas only $32.5 \%$ of the IPT patients did so (see Table 1). These results indicate that BDI-II scores around large reductions in symptoms tended to fluctuate more in IPT, thereby resulting in fewer patients who met the full set of criteria for sudden gains.

\subsection{Baseline predictors of sudden gains}

Univariate logistic regressions identified 4 variables with a promising association with the dependent variable (see data supplement I). The selected variables were entered into a multivariate regression model (see Table 4 for full model), and non-significant variables were eliminated in a manual backward procedure until the model contained only the two significant predictors of sudden gains. As shown in Table 4, patients were more likely to experience a sudden gain if they entered treatment with a higher Quality of Life Utility score or if comorbidity on Axis I was absent. Tests of the interactions of these two variables with treatment condition were non-significant (all interactions $p>.55$ ).

\subsection{Sudden gains and outcome}

Patients with sudden gains showed more improvement during treatment as compared to those without sudden gains, and consequently reported lower post-treatment BDI-II scores (baseline corrected estimated means of $10.7(\mathrm{SE}=1.7)$ vs. $18.5(\mathrm{SE}=1.2) ; F(1$, $106)=13.12, p<.001)$. Furthermore, at the end of the treatmentphase, $44.4 \%$ of the sudden gain patients met criteria for remission (BDI-II $<9$ ) versus $25.0 \%$ of those without sudden gains $\left(\chi^{2}\right.$ $(1)=4.21, p=.040)$. A similar pattern emerged at 5 months FU (mean baseline corrected BDI-II scores of 9.7 ( $\mathrm{SE}=2.1$ ) vs. 18.6 $(\mathrm{SE}=1.5) ; F(1,101)=11.60, p<.01$; remission rates of $54.3 \% \mathrm{vs}$. $\left.33.8 \% ; \chi^{2}(1)=4.00, p=.045\right)$. The additional model that controlled for both baseline- and post-treatment severity, did not show a significant difference between the two patient groups (difference of 1.7 BDI-II points $(\mathrm{SE}=1.6)$ in favor of patients with sudden gains; $F(1,101)=1.14, p=.288)$. Baseline corrected depression severity scores for patients with and without sudden gains at treatment termination and at 5 months FU stratified by treatment condition are displayed in Table 5. Within both treatment groups, differences between gainers and non-gainers were significant at both timepoints (all p's <.04). As can be seen in the table, BDI-II scores of patients with sudden gains are fairly similar in CT and IPT, but it appears that they differ between conditions among patients who did not experience a sudden gain. However, tests of the gain* condition interactions were non-significant at both post-treatment $(F(1,106)=0.51, p=.476)$ and at 5 months $F U(F(1,101)=1.41$, 
Table 3

Comparison of results between relevant studies examining sudden gains in individual CT and IPT for adult unipolar depression.

\begin{tabular}{|c|c|c|c|c|c|c|c|c|}
\hline & \multicolumn{6}{|l|}{ Cognitive Therapy } & \multicolumn{2}{|c|}{ Interpersonal Psychotherapy } \\
\hline & $\begin{array}{l}\text { Tang \& DeRubeis } \\
\text { 1999b }\end{array}$ & $\begin{array}{l}\text { Tang et al., } \\
2005\end{array}$ & $\begin{array}{l}\text { Hardy et al., } \\
2005\end{array}$ & $\begin{array}{l}\text { Busch et al., } \\
2006\end{array}$ & $\begin{array}{l}\text { Tang et al., } \\
2007\end{array}$ & $\begin{array}{l}\text { Current } \\
\text { study* }\end{array}$ & $\begin{array}{l}\text { Kelly et al., } \\
2007\end{array}$ & $\begin{array}{l}\text { Current } \\
\text { study* }\end{array}$ \\
\hline - Sessions, $n$ & 20 & $12-20$ & $8-20$ & 20 & 20 & $16-20$ & 12 & $16-20$ \\
\hline - Sample Size, $n$ & 61 & 46 & 76 & 38 & 60 & 64 & 185 & 53 \\
\hline - Patients with SG's, \% & $39.3 \%$ & $43.0 \%$ & $41.0 \%$ & $42.1 \%$ & $40.0 \%$ & $42.2 \%$ & $33.5 \%$ & $24.5 \%$ \\
\hline - Magnitude, BDI-II, M (SD) & $11.2(4.4)$ & $10.2(2.8)$ & $13.3(5.0)$ & $9.3(2.9)$ & 11 & $10.3(3.7)$ & $13.0(5.3)$ & $12.4(5.3)$ \\
\hline - Pre-gain session, $m$ & 5 & 8 & 5 & 10 & 5 & 9 & - & 9 \\
\hline - Patients with reversals, \% & $16.7 \%$ & $40.0 \%$ & $32.3 \%$ & $43.8 \%$ & $37.5 \%$ & $22.2 \%$ & $53.2 \%$ & $15.4 \%$ \\
\hline - Patients that maintained SG's, \% & $32.8 \%$ & $26.1 \%$ & $27.6 \%$ & $23.7 \%$ & $25.0 \%$ & $32.8 \%$ & $15.7 \%$ & $20.8 \%$ \\
\hline
\end{tabular}

SG = Sudden Gain; BDI-II = Beck Depression Inventory-II; * = original sudden gain criteria defined by Tang and DeRubeis (1999b).

Table 4

Full and final model of the multivariate logistic regression analysis $(n=117)$.

\begin{tabular}{|c|c|c|c|}
\hline & OR & $95 \% \mathrm{CI}$ & $p$ \\
\hline \multicolumn{4}{|l|}{ Full model } \\
\hline - Quality of Life Utility score (EuroQol) ${ }^{1}$ & 1.20 & $.98-1.47$ & .08 \\
\hline - Absence of Comorbid Axis I disorder & 2.88 & $1.22-6.76$ & .02 \\
\hline - Level of Impairment: Physical Functioning & 1.01 & $.99-1.03$ & .37 \\
\hline - Hopelessness: feelings about the future & 1.33 & $.98-1.78$ & .06 \\
\hline \multicolumn{4}{|l|}{ Final Model* } \\
\hline - Quality of Life Utility score (EuroQol) ${ }^{1}$ & 1.24 & $1.04-1.49$ & .02 \\
\hline - Absence of Comorbid Axis I Disorder & 2.64 & $1.15-6.08$ & .02 \\
\hline
\end{tabular}

Dependent Variable $=$ Gainer (yes/no); ${ }^{1}=$ Adjusted score (EuroQol Utility ${ }^{*} 10$ ): * = Order of elimination: 1) Level of impairment: Physical functioning; 2) Hopelessness: feelings about the future.

$p=.238)$. The model corrected for post-treatment severity at 12 months yielded similar results $(F(1,101)=1.30, p=.256)$.

\subsection{Sudden gains and response to treatment}

At the end of the treatment phase, 81 of the 117 patients in CT or IPT met the criterion for response (a decrease of at least 9 BDI-II points over the course of treatment). Response rates were similar for CT and IPT (70.3\% vs. 67.9\%; $\chi^{2}(1)=.08, p=.781$ ). Among the 81 responders, 32 patients (39.5\%) experienced a sudden gain. Overall, responders whose improvement included a sudden gain were found to experience significantly better post-treatment outcomes compared to responders who did not experience a sudden gain (baseline corrected BDI-II scores of 9.3 ( $\mathrm{SE}=1.3$ ) vs. 13.5 ( $\mathrm{SE}=1.1), F$ $(1,79)=6.53, p=.013)$. At 5 months FU the difference between the two groups was somewhat larger (baseline corrected BDI-II scores of $8.2(\mathrm{SE}=1.6)$ for sudden gain responders vs. $13.7(\mathrm{SE}=1.3)$ for non-sudden gain responders; $F(1,75)=6.82, p=.011$ ). However, after controlling for the post-treatment score as well, the difference at 12 months was not statistically significant anymore ( $F(1$, $75)=2.01, p=.160)$. Fig. 1 gives the baseline corrected BDI-II scores in CT and IPT at post-treatment and 5 months FU for sudden-gainresponders and non-sudden-gain-responders. Tests of the gain*condition interactions were non-significant at both time points (all interactions $p>.08$ ).

\subsection{Secondary analyses: effects of the duration of the between- session interval}

Excluding sudden gains that occurred outside the acceptable between-session interval reduced the number of patients that were identified as sudden gainers from 27 to 19 (29.7\%) in CT and 13 to 8 (15.1\%) in IPT. The difference in rates between the two conditions became a non-significant trend $\left(\chi^{2}(1)=3.47, p=.062\right)$. Characteristics (e.g., magnitude, timing and reversals) and baseline predictors of these sudden gains were similar to those observed when we allowed for longer intervals between the gains (see data supplement II). Furthermore, contrasting patients with sudden gains that occurred within the 14-day interval ( $n=27$ ) to sudden gainers with gains that were recorded on a longer between-session interval ( $n=13$ ), showed no significant differences on outcome, both at post-treatment (adjusted mean difference of 0.8 BDI-II points, $\mathrm{SE}=3.8, p=.848$ ) and 5 months FU (adjusted mean difference $=-1.9, \mathrm{SE}=4.5, p=.676$; also see data supplement II).

\section{Discussion}

The present study was the first to examine the occurrence, baseline predictors and clinical impact of sudden gains in a randomized comparison of individual CT and IPT for adult depression. Among those who met inclusion criteria for our analyses, a higher proportion of patients in CT experienced sudden gains, as compared to patients allocated to IPT. Group differences seemed mainly to be driven by the $c$ criterion, which indexes the stability of the drop. The difference in rates between the two conditions became a non-significant trend when only gains that occurred within a between-session interval of less than 14 days were considered. In both conditions, sudden gains were predicted by baseline quality of life score and the absence of axis-I comorbidity, suggesting that relatively "good health" at baseline predicts sudden

Table 5

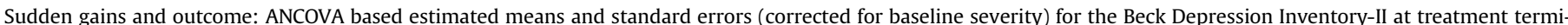
nation and 5 months FU for patients with and without Sudden Gains stratified according to treatment condition ( $n=117)$.

\begin{tabular}{|c|c|c|c|c|}
\hline & \multicolumn{2}{|c|}{ Cognitive Therapy $(n=64)$} & \multicolumn{2}{|c|}{ Interpersonal Psychotherapy $(n=53)$} \\
\hline & Gainers $(n=27)$ & Non-gainers $(n=37)$ & Gainers $(n=13)$ & Non-gainers $(n=40)$ \\
\hline 7 months & $10.4(1.9)$ & $16.8(1.6)$ & $9.7(3.5)$ & $20.7(1.9)$ \\
\hline 12 months & $9.8(2.2)$ & $15.7(1.9)$ & $7.4(4.5)$ & $21.9(2.4)$ \\
\hline
\end{tabular}

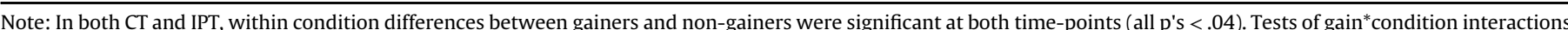

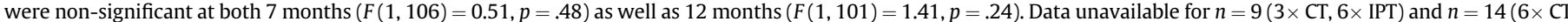
and $8 \times$ IPT) patients at 7 and 12 months respectively. All scores corrected for baseline severity (BDI-II). 


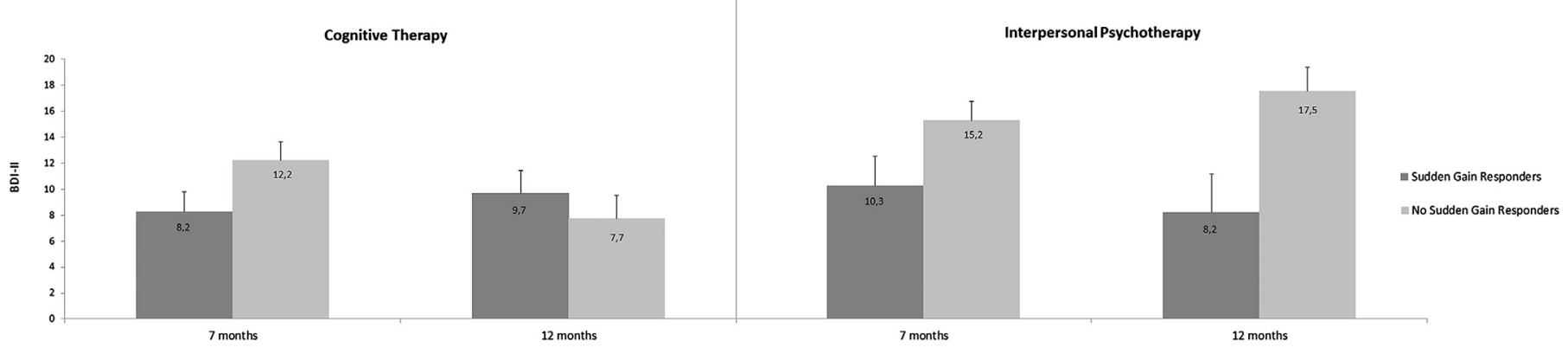

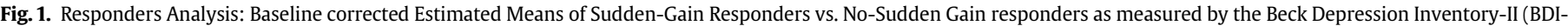

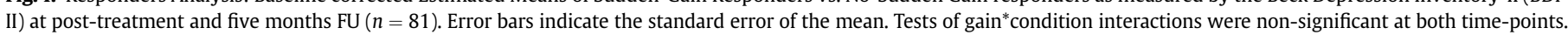

gains. Furthermore, in line with what is reported in the literature (Aderka et al., 2011), patients with sudden gains were less depressed at post-treatment than were those without sudden gains, and they remained so 5 months after treatment termination. Considering only those patients whose sudden gains occurred over a between-session interval of only one or two weeks, which reduced the number of patients identified as sudden gainers, did not affect the CT/IPT ratio, the characteristics and predictors of sudden gains, or the advantage observed in outcome for those with sudden gains.

The frequency of sudden gains observed in the CT condition is within the range reported in other relevant studies in the field (see Table 3 for an overview). Sudden gain rates observed in the IPT condition would appear to be low when compared with those obtained in a previous study of IPT by Kelly et al. (2007). However, high reversal rates were reported in the Kelly et al. study, whereas the reversal rates in the present study indicate that the vast majority of sudden gains were maintained throughout treatment. When taking this into account and thus focusing only on those patients who maintained their sudden gains throughout therapy, the proportion of sustained sudden gains in the present study was no longer lower than Kelly et al.'s (see Table 3). The difference in the frequency of sudden gains in CT versus IPT that was found in the current study initially seems not to be in line with findings of Bohn et al. (2013) who examined sudden gains in CT vs. IPT for social anxiety disorder and did not find a statistically significant frequency difference between the two groups. It should be noted that Bohn et al. included a smaller sample $(n=67)$ and that the proportion of patients with sudden gains was relatively small $(n=15$, corresponding with $22.0 \%$ of the sample). As a result, the power to test for between-group differences was lower. Furthermore, though not statistically different, there were twice as many patients with sudden gains in CT $(n=10)$ compared to IPT $(n=5)$. This is in line with the CT/IPT ratio that was found in the current study $(27 / 13)$. The fact that none of the pre-treatment measures that address CTor IPT-relevant processes predicted the occurrence of sudden gains is in line with findings of Hunnicutt-Ferguson et al. (2012) and Kelly et al. (2005), who also did not find a relation between pretreatment variables and the occurrence of sudden gains. These findings lend further support to the idea that the causes of sudden gains are not likely to be found with measures obtained long before the gain occurs, but rather with assessments of processes that occur over the course of therapy, in close temporal proximity to the gain.

\subsection{Cognitive Therapy vs. Interpersonal Psychotherapy}

Although our results are in line with those obtained in other studies, the question that remains unanswered is why sudden gains are less likely to occur in IPT. One explanation could be that these large and sudden symptom improvements are indeed mainly driven by cognitive processes (as suggested by Tang \& DeRubeis, 1999b). Although non-cognitive interventions may also lead to cognitive changes (e.g. Weissman, Markowitz, \& Klerman, 2000), the emphasis on cognitive processes in CT may lead to more sudden gains in comparison to therapeutic approaches such as IPT that rely more on non-cognitive interventions. However, even though we see merit in this explanation, we think it is unlikely that this fully explains our findings. Results indicate that the difference between the two treatment modalities emerged not because CT facilitated a higher number of large symptom improvements between two consecutive sessions, relative to IPT, but rather was due to the relative lack of stability in the pre- and post-gain sessions in IPT. This points to possible differences in the therapeutic process in $\mathrm{CT}$ and IPT. Because IPT focuses on solving difficulties in an interpersonal context, it may be more common in IPT for progress to be affected by the vagaries of patient's interactions with others. For example, to solve a problem or reach a specific goal in IPT the patient will likely engage with significant others. Whether these interpersonal encounters will lead to improvement in depressive symptoms depends on experiences during and the outcome of these encounters. As a result, levels of depressive symptoms may be more unstable during IPT than during CT. Second, CT includes homework between sessions, whereas IPT does not. This might promote the consolidation of the skills that are learned in treatment and therefore to more stable symptom improvement. A third possibility is that differences in therapists' attitude affect the stability of session-by-session BDI-II scores. The CT therapist takes a directive role as he or she leads the patients through the protocol, whereas the IPT therapist is merely a supportive ally as he or she helps the patient explore options in an interpersonal context, thus allowing for more instances of trial and error. These possibilities should be explored in future research on the effects of CT and IPT for depression.

\subsection{Methodological considerations and recommendations for future research}

The current study was novel in that it allowed for a direct comparison between CT and IPT in the occurrence and nature of sudden gains in a large sample of adult depressed outpatients. Furthermore, it is the second study of sudden gains in IPT. By using a more heterogeneous group than Kelly et al. (2007) (males as well as females with first-episode and recurrent depressions) we increased the generalizability of previous findings. In addition, the inclusion of a separate responders analysis diminished uncertainty as to whether patients who experienced sudden gains were nothing more than treatment responders. Moreover, by explicitly examining the impact of the duration of the between-session interval, we paid attention to an important aspect of sudden gains that has been largely overlooked in the literature. To our knowledge 
there is only one other study that explicitly ensured the suddenness of the gain by excluding those observed over intervals that were considered too long (Kelly et al., 2007).

One limitation of the current study is that the percentage of patients who met criteria for sudden gains may have been too low, especially in IPT and after eliminating sudden gains that occurred outside the acceptable between-session interval of 14 days, to support powerful tests of potential differences between the two treatments in predictors and the relation of sudden gains with short and long-term measures of outcome. Second, our study was powered to detect (long-term) clinical differences between CT and IPT in terms of absolute BDI-II scores (Lemmens et al., 2011, 2015). This may have limited the power of the current analyses. A third limitation is that although the findings of pre-treatment variables that predict sudden gains add to an understanding of this phenomenon, they do not speak to the processes that occur over the course of therapy and elicit sudden gain.

Future studies should therefore examine the differences between CT and IPT more closely, both in the short-term and in the long run, paying special attention to the impact of the duration of the between-session interval at which sudden gains are recorded. Furthermore, studies are needed that focus on therapy-specific as well as common factors that precede sudden gains by using observer-rated measures to capture relevant phenomena in the pre-gain session. A better understanding of sudden gains and their associated factors may provide insight into the mechanisms of change in psychotherapy and, further, may suggest areas for increased therapeutic focus and thereby contribute to the improvement of treatments for adults suffering from depression.

\section{Conflict of interest}

The authors declare that they have no competing interests.

\section{Funding}

This research was funded by the Research Institute of Experimental Psychopathology (EPP), The Netherlands, and the Academic Community Mental Health Centre (RIAGG) in Maastricht, the Netherlands.

\section{Acknowledgments}

We wish to acknowledge the contribution of participating patients and therapists at RIAGG Maastricht. Furthermore, we gratefully thank Annie Raven and Annie Hendriks for their assistance during the study, and Tony Tang for his input and advice with regard to this paper.

\section{Appendix A. Supplementary data}

Supplementary data related to this article can be found at http:// dx.doi.org/10.1016/j.brat.2015.12.014.

\section{References}

psychological treatments of anxiety and depression: a meta-analysis. Journal of Consulting and Clinical Psychology, 80(1), 93-101.

Beck, A. T., Rush, A. J., Shaw, B. F., \& Emery, G. (1979). Cognitive therapy of depression. New York, NY: Guilford Press.

Beck, A. T., Steer, R., \& Brown, G. K. (1996). Beck depression inventory II: Manual. Boston, MA: Hartcourt Brace.

Bohn, C., Aderka, I. M., Schreiber, F., Stangier, U., \& Hofmann, S. G. (2013). Sudden gains in cognitive therapy and interpersonal therapy for social anxiety disorder Journal of Consulting and Clinical Psychology, 81(1), 177-182.

Busch, A. M., Kanter, J. W., Landes, S. J., \& Kohlenberg, R. J. (2006). Sudden gains and outcome: a broader temporal analysis of cognitive therapy for depression. Behavior Therapy, 37(1), 61-68.

Cuijpers, P., Geraedts, A. S., van Oppen, P., Andersson, G., Markowitz, J. C., \& van Straten, A. (2011). Interpersonal psychotherapy for depression: a meta-analysis. American Journal of Psychiatry, 168, 581-592.

Elkin, I., Shea, M. T., Watkins, J. T., \& Imber, S. D. (1989). National institute of mental health treatment of depression collaborative research program: general effectiveness of treatments. Archives of General Psychiatry, 46(11), 971-982.

First, M. B., Spitzer, R. L., Gibbon, M., \& Williams, J. B. W. (1997). Structured clinical interview for DSM-IV Axis I disorders (SCID-I). New York, NY: Biometrics Research Department New York State Psychiatric Institute.

Hardy, G. E., Cahill, J., Stiles, W. B., Ispan, C., Macaskill, N., \& Barkham, M. (2005). Sudden gains in cognitive therapy for depression: a replication and extension. Journal of Consulting and Clinical Psychology, 73(1), 59-67.

Hollon, S. D., DeRubeis, R. J., Evans, M. D., Wiemer, M. J., Garvey, M. J., Grove, W. M. et al. (1992). Cognitive therapy and pharmacotherapy for depression: singly and in combination. Archives of General Psychiatry, 49(10), 774-781.

Hollon, S. D., Thase, M. E., \& Markowitz, J. C. (2002). Treatment and prevention of depression. Psychological Science and Public Interest, 3, 39-77.

Hunnicutt-Ferguson, K., Hoxha, D., \& Gollan, J. (2012). Exploring sudden gains in behavioral activation therapy for major depressive disorder. Behaviour Research and Therapy, 50(3), 223-230.

Kazdin, A. E., \& Nock, M. K. (2003). Delineating mechanisms of change in child and adolescent therapy: methodological issues and research recommendations. Journal of Child Psychology and Psychiatry, 44(8), 1116-1129.

Kelly, M. A. R., Cyranowski, J. M., \& Frank, E. (2007). Sudden gains in interpersonal psychotherapy for depression. Behaviour Research and Therapy, 45(11), $2563-2572$.

Kelly, M. A. R., Roberts, J. E., \& Ciesla, J. A. (2005). Sudden gains in cognitive behavioral treatment for depression: when do they occur and do they matter? Behaviour Research and Therapy, 43(6), 703-714.

Klerman, G. L., Weissman, M. M., Rounsaville, B. J., \& Chevron, E. S. (1984). Interpersonal psychotherapy for depression. New York, NY: Basis Books.

Kraemer, H. C., Wilson, G. T., Fairburn, C. G., \& Agras, W. S. (2002). Mediators and moderators of treatment effects in randomized clinical trials. Archives of General Psychiatry, 59, 877-883.

Laurenceau, J.-P., Hayes, A. M., \& Feldman, G. C. (2007). Some methodological and statistical issues in the study of change processes in psychotherapy. Clinical Psychology Review, 27(6), 682-695.

Lemmens, L. H. J. M., Arntz, A., Peeters, F. P. M. L., Hollon, S. D., Roefs, A., \& Huibers, M. J. H. (2011). Effectiveness, relapse prevention and mechanisms of change in cognitive therapy vs. interpersonal therapy for depression: study protocol for a randomised controlled trial. Trials, 12, 150-162.

Lemmens, L. H. J. M., Arntz, A., Peeters, F. P. M. L., Hollon, S. D., Roefs, A., \& Huibers, M. J. H. (2015). Clinical effectiveness of cognitive therapy vs. Interpersonal psychotherapy for depression: results of a randomised controlled trial. Psychological Medicine, 45, 2095-2110.

Tang, T. Z., \& DeRubeis, R. J. (1999a). Reconsidering rapid early response in cognitive behavioral therapy for depression. Clinical Psychology: Science and Practice, 6(3), 283-288.

Tang, T. Z., \& DeRubeis, R. J. (1999b). Sudden gains and critical sessions in cognitivebehavioral therapy for depression. Journal of Consulting and Clinical Psychology, 67(6), 894-904.

Tang, T. Z., DeRubeis, R. J., Beberman, R., \& Pham, T. (2005). Cognitive changes, critical sessions, and sudden gains in cognitive-behavioral therapy for depression. Journal of Consulting and Clinical Psychology, 73(1), 168-172.

Tang, T. Z., DeRubeis, R. J., Hollon, S. D., Amsterdam, J., \& Shelton, R. (2007). Sudden gains in cognitive therapy of depression and depression relapse/recurrence. Journal of Consulting and Clinical Psychology, 75(3), 404-408.

Vittengl, J. R., Clark, L. A., \& Jarrett, R. B. (2005). Validity of sudden gains in acute phase treatment of depression. Journal of Consulting and Clinical Psychology, 73(1), 173-182.

Weissman, M. M., Markowitz, J. C., \& Klerman, G. L. (2000). Comprehensive guide to interpersonal psychotherapy. New York, NY: Basic Books. 